Journal of Computer Science 7 (10): 1605-1611, 2011

ISSN 1549-3636

(C) 2011 Science Publications

\title{
Application of Neuro-Fuzzy Techniques for Solar Radiation
}

\author{
${ }^{1}$ W.A. Rahoma, ${ }^{2}$ U. Ali Rahoma and ${ }^{3}$ A.H. Hassan \\ ${ }^{1}$ Department of Astronomy, Cairo University \\ ${ }^{2,3}$ Department of National Research Institute of Astronomy and Geophysics, Egypt
}

\begin{abstract}
Problem statement: The prediction is very useful in solar energy applications because it permits to estimate solar data for locations where measurements are not available. The developed artificial intelligence models predict the solar radiation time series more effectively compared to the conventional procedures based on the clearness index. Approach: The forecasting ability of some models could be further enhanced with the use of additional meteorological parameters. After having simulated many different structures of neural networks and trained using measurements as training data, the best structures were selected in order to evaluate their performance in relation with the performance of a neuro-fuzzy system. As the alternative system, ANFIS neuro-fuzzy system was considered, because it combines fuzzy logic and neural network techniques that are used in order to gain more efficiency. ANFIS is trained with the same data. Results: The comparison and the evaluation of both of the systems were done according to their predictions, using several error metrics. Fuzzy model was trained using data of daily solar radiation recorded on a horizontal surface in National Research Institute of Astronomy and Geophysics, Helwan, Egypt (NARIG) at ten years (1991-2000). Conclusion: The predicting conclusion indicated that the TS fuzzy model gave a good accuracy of approximately $96 \%$ and a root mean square error lower than $6 \%$.
\end{abstract}

Key words: Forecasting ability, neural network, neuro-fuzzy system, error metrics, Wang-Mendel Method (WM), solar radiation, Fuzzy Predictor (FP), Truth Degree (TD), alternative system, Auto-Regressive Moving Average (ARMA), Auto-Regressive (AR)

\section{INTRODUCTION}

The predicting results indicate that the TS fuzzy model gives a good accuracy of approximately $96 \%$ and a root mean square error lower than 6\%. Fuzzy rule based approach is widely accepted as a promising tool because it uses linguistic terms, it is able to deal with nonlinear problems and can perform predictions at high speed. For those reasons, the problem we address here describes the on-going research effort that takes place to shed light on the applicability of using fuzzy rules to estimate the solar radiation irradiance time series in the plain areas of Egypt. Chen (1994) first constructed fuzzy relationship with 'IF-THEN' rule group between fuzzy time series. And then, many researches extend and modify Chen's model and still construct fuzzy relationship with rule based (Ahmed and Isa, 2009; Michael, 2010; Efendioglu and Karabulut, 2009; Alexandros et al., 2010; Kar and Kundu, 2010; Abd Elaal et al., 2010). On forecasting step, they all use $100 \%$ match method to classify the pattern of fuzzy time series, like classify category data. However, fuzzy set and fuzzy time series are not category data. The similarity measure between fuzzy time series is need on pattern recognition for fuzzy time series forecasting model. In this study we represent a fuzzy predictive Solaimani, 2009; Dastorani et al., 2010) method for global prediction which learns an input output mapping (Ismail et al., 2010; Anton et al., 2009; Saleh et al., 2009; Al-Suhaibani et al., 2010; Effendi et al., 2010; Eslamian et al., 2009; Kang and Jin, 2010; Podeh et al., 2009).

The WM method was one of the first methods to design fuzzy systems from data. The meteorological variables including air temperature, solar radiation, wind speed and relative humidity were considered daily. The $\mathrm{R}^{2}$ of ANNs and SVMs models were obtained 0.92 and 0.96 , respectively; whereas the efficiency of ANNs and SVMs models were 0.83 and 0.91, respectively. Both ANNs and SVMs approaches work well for the data set used in greenhouse condition, but the SVMs model works better in comparison with the ANNs model.

In this study, we propose a new similarity measure between fuzzy sets and between fuzzy time series and its application to pattern recognition for forecasting and outperform than $100 \%$ match method of category data in fuzzy time series forecasting model. 
Data based, on many different scientific efforts in order to realize better results in the domain of forecasting meteorological parameters. Temperature and solar radiation forecasting constitutes a very crucial issue for different scientific areas as well as for many different aspects of everyday life. It has to be mentioned that the meteorological data used, come from the NRIAG, Helwan. The data obtained were hourly measurements for the period 1991-2000 and were measured in $\mathrm{w} \mathrm{m}^{2}$. After observing the data it's obvious that apart from the logical values. They constitute measurement errors and have to be replaced.

The object is to create the mean daily solar radiation time series without error measurements in order to use it to the prediction systems.

\section{MATERIALS AND METHODS}

Fuzzy Predictor (FP): The method (Yedjour et al., 2011; Mahi and Izabatene, 2011;Guang et al., 2009; Alsaade, 2010) , for prediction system design presents three characteristics: simplicity, a one-pass operation on the numerical input-output pairs to extract the rules and fast computational time. Suppose we are given $\mathrm{N}$ inputoutput samples:

$$
\left(\mathrm{x}_{1}^{(\mathrm{p})}, \mathrm{x}_{2}^{(\mathrm{p})}, \ldots, \mathrm{x}_{\mathrm{M}}^{(\mathrm{p})} ; \mathrm{y}^{(\mathrm{p})}\right) \mathrm{y}^{(\mathrm{p})} \in \mathrm{R} \mathrm{p}=1,2, \ldots, \mathrm{N}
$$

Where:

$\mathrm{x}_{\mathrm{i}}=$ Inputs

$\mathrm{M}=$ The number of the inputs

$\mathrm{y}=$ The output

This method consists of the following five steps as shown in Fig. 1. ANFIS is a technique for automatically tuning (Back propagation algorithm) TS-type fuzzy inference system based on some collection of input-output data. The ANFIS predictor uses first-order TS-type systems, single output derived by weighted defuzzification and membership function type that is a generalized bell curve (MATLAB). Another method for solving forecasting tasks is the combination of neural network predictors called committee machine Aggarwal et al., 2005; Babuska, 1996; Chen, 1994; Huarng et al., 2007; Li, and Cheng, 2009; Pratumsuwan et al., 2010). The proposed committee machine is a static structure. In this technique the predictors are combined by means of a mechanism that does not involve the input signal. The outputs of four different predictors are nonlinearly combined to produce an optimum prediction as shown in Fig. 2. Here, the nonlinear mechanism is the neuro-fuzzy system ANFIS which is trained by the output data from predictors. For simplicity, the NNP will be referred to as IL-HL-OL network, corresponding to the number of nodes in each layer (IL: Input Layer, HL: Hidden layer and OL: Output layer). In iteration (time step) $\mathrm{n}$, the nth training example is presented to the network. The symbol $Z_{i}(n)$ refers to the prediction signal appearing at the output of $\mathrm{NNP}_{\mathrm{i}}$. The symbol $\mathrm{Y}(\mathrm{n})$ refers to the final prediction at iteration $\mathrm{n}$.

1st step: Divide the input and output spaces into fuzzy regions: We consider that the input $x_{i}$ and the output $\mathrm{y}$ lie in the domain intervals $\left[\mathrm{x}_{\mathrm{i} \mid \min }, \mathrm{x}_{\mathrm{i} \mid \max }\right]$ and $\left[y_{\min }, y_{\max },\right]$ respectively. We divide each interval into $2 z+1$ fuzzy region and assign each region a symmetrical triangular fuzzy set. Of course, other shapes of membership functions are possible.

2nd step: Data-generated fuzzy rules: From the training set, take the $\mathrm{m}^{\text {th }}$ numerical data $\operatorname{pair}\left(\mathrm{x}_{1}^{(\mathrm{m})}, \mathrm{x}_{2}^{(\mathrm{m})}, \ldots, \mathrm{x}_{\mathrm{M}}^{(\mathrm{m})} ; \mathrm{y}^{(\mathrm{m})}\right)$. For each data pair calculates their respective membership grades in the attributed fuzzy sets. Next, choose for each variable their highest membership degree from the respective grades. Now, a rule from the $\mathrm{m}$ training pair is obtained:

$$
\begin{aligned}
& \mathrm{R}^{\mathrm{m}}: \mathrm{IF}_{1}^{\mathrm{m}} \text { is } \mathrm{A}_{1}^{\mathrm{m}} \text { AND...AND } \mathrm{x}_{\mathrm{M}}^{\mathrm{m}} \\
& \text { is } \mathrm{A}_{\mathrm{M}}^{\mathrm{m}} \text { THEN } \mathrm{y}^{\mathrm{m}} \text { is } \mathrm{C}^{\mathrm{m}}
\end{aligned}
$$

where, $\mathrm{A}_{\mathrm{i}}^{\mathrm{m}}$ and $\mathrm{C}^{\mathrm{m}}$ are fuzzy sets that attributed in the condition and conclusion parts of the rule and $\mathrm{m}$ is the index of the rule. Especially, we define $\mathrm{l}_{\mathrm{i}}(\mathrm{i}=1, \ldots, \mathrm{M})$ fuzzy sets $A_{i}^{q}, q=1, \ldots l_{i}$ for each input $x_{i}$ where $1_{0}$ represents the number of membership functions in the output space.

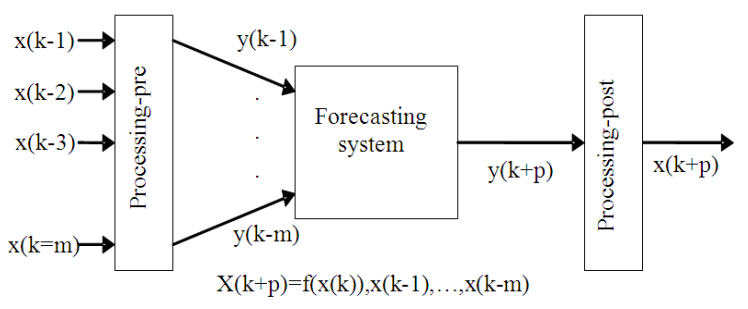

Fig. 1: Basic structure of the forecasting system

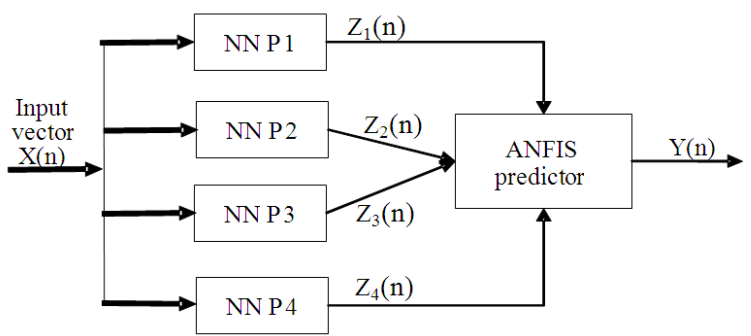

Fig. 2: A typical architecture of a committee machine based 
The fuzzy set $A_{i}^{m}$ is one of the $A_{i}^{q}$ 's. Generally, in real applications we give, in the fuzzy sets linguistic names, like "big", "very positive".

3rd step: Assign a degree to each rule: As there are usually many data pairs and therefore many rules generated, there is high probability of conflict. That is, rules which have the same IF part and a different then part. To resolve this problem is to assign a Truth Degree (TD) to each rule and accept only the rule that has the largest truth degree. We use the following product strategy:

$\mathrm{TD}^{(\mathrm{m})}=\mu_{\mathrm{A}_{\mathrm{m}}^{\mathrm{m}}}\left(\mathrm{x}_{1}^{(\mathrm{m})}\right) \cdots \cdot \mu_{\mathrm{A}_{\mathrm{M}}^{\mathrm{m}}}\left(\mathrm{x}_{\mathrm{M}}^{(\mathrm{m})}\right) \cdot \mu_{\mathrm{C}^{\mathrm{m}}}\left(\mathrm{y}^{(\mathrm{m})}\right)$

4th step: Create a combined fuzzy rule base: The maximum number of rules that can be generated is $1_{1}$. $1_{2} \cdot \ldots \cdot l_{\mathrm{M}}$. From the 3 rd step the reduction of the number of rules is achieved. The generated rules determine a combined fuzzy rule base.

5th step: Determine a mapping based on the combined fuzzy rule base: Determine the overall continuous fuzzy predictive model. Using the combined rule base with $\mathrm{K}$ fuzzy rules, the product inference engine, the singleton fuzzifier and the center-average defuzzifier, the following fuzzy system is obtained Chen (1997):

$$
y=f(x)=\frac{\sum_{j=1}^{K} y_{c}^{j}\left(\prod_{i=1}^{M} \mu_{A_{i}^{j}}\left(x_{i}\right)\right)}{\sum_{j=1}^{K}\left(\prod_{i=1}^{M} \mu_{A_{i}^{j}}\left(x_{i}\right)\right)}
$$

Where, $y_{c}^{j}$ is the centre of $C^{j}$. The output variable $y$ is based on the inputs $\left(\mathrm{x}_{1}, \mathrm{x}_{2}, \ldots, \mathrm{x}_{\mathrm{M}}\right)$. Training methods object is to minimize the mean square error between the predictions and the real values. In order to find the most appropriate training algorithm, there was created a small neural network 3-10-1, with sigmoid activation function in the nodes of the hidden layer and it was simulated for normalized data in the region 0.1-0.9 for 13 different algorithms that appear in the neural toolbox of MATLAB. Five error metrics were used in order to choose the most efficient algorithm: MSE, RMSE, AME, NDEI, $\rho$. The algorithms used are the following:

\section{Error metrics:}

$$
\begin{aligned}
& \text { MSE }=\frac{1}{n} \sum_{k=1}^{n}(x(k)-\hat{x}(k))^{2} \\
& \text { RMSE }=\sqrt{\frac{1}{n} \sum_{k=1}^{n}(x(k)-\hat{x}(k))^{2}} \\
& \text { AME } \left.=\frac{1}{n} \sum_{k=1}^{n} \mid x(k)-\hat{x}(k)\right) \mid \\
& \text { NDEI }=\frac{\text { RMSE }}{\sigma}=\sqrt{\frac{\sum_{k=1}^{n}(x(k)-\hat{x}(k))^{2}}{\sum_{k=1}^{n} x^{2}(k)}} \\
& \rho=\frac{\sum_{k=1}^{n}(x(k)-\bar{x}) \cdot(\hat{x}(k)-\overline{\hat{x}})}{\sqrt{\sum_{k=1}^{n}(x(k)-\bar{x})^{2} \cdot \sum_{k=1}^{n}(\hat{x}(k)-\overline{\hat{x}})^{2}}}
\end{aligned}
$$

Where:

$\mathrm{x}(\mathrm{k})=$ The real value in time instance $\mathrm{k}$

$\mathrm{x}(\mathrm{k})=$ The prediction of the model

$\mathrm{N}=$ Number of test data used for the prediction

It has to be mentioned that the most characteristic error criterion showing the quality of prediction was proved be the correlation coefficient criterion $(\rho)$. As the prediction improves, $\rho$ is getting close to 1 .

Neural networks training: After having trained and tested all the different cases and structures of neural networks with the different in normalization and type meteorological time-series, they have been compared according to the above error criteria in order to result in the most suitable neural predictor's structure for every different time-series:

- In the beginning there were chosen the best four neural networks for every different type of normalization

- Next there were chosen the best four neural network predictors for every different type of meteorological time-series in order to use them to more complex systems like neural network committee machines

- Finally there was made choice of the best neural network predictor for every different time-series in order to be able to compare its results with ANFIS or any other system created

The best neural network predictors for every different time-series using Sigmoid Activation Function $(\mathrm{SAF})$ are introduced.

Mean daily solar radiation: 5-15-1, normalized in the range 0.1-0.9, using SAF. 
Mean daily temperature: $5-10-1$, normalized in the range 0.1-0.9, using SAF.

'Best' neural network predictors Vs ANFIS: For the training and testing of data there was created a first TS type system consisting of 7 inputs. The combination of fuzzy logic with neural networks proved to have very good results in the daily solar radiation and temperature forecasting. Below there is presented a comparison between the 'best' N.N. predictors and ANFIS, for the four different time series, using as criterion the metric that proved to be the most accurate, which is the correlation coefficient $(\rho)$ as shows in Table 1. For the global prediction scheme we split the collected data into two categories. The training set consists of the temperature of the first five years (1991-1995), while the test set includes the remaining five years (19962000). The choice of four inputs to form the TS method is case-dependent. For the numerical fuzzy approach 125 rules were obtained. In the table of comparison the computational time has also been recorded.

Prediction results with NNP: The main object is to create many different structures of neural network predictors and train and test them with the meteorological data available in order to conclude the best and more efficient topology for forecasting solar radiation and temperature. For the meteorological time series used in this study, one hidden layer is appropriate and sufficient. The following cases of structures of neural networks have been created and simulated: Inputs: 2,3,5,7 previous daily measurements for the 12 different time series created (real and normalized data), Number of hidden layers: 1, Number of nodes of hidden layer: 2,3,5,10,15, Output: 1 (One day prediction), Activation Functions used in neurons: Hidden layer: sigmoid, linear, Output layer: linear Babuska (1996).

After having trained and tested all the different cases and structures of neural networks with the different in normalization and type meteorological time-series, they have been compared according to the above error criteria in order to result in the most suitable neural predictors' structure for every different time-series.

In the beginning, the best four neural networks for every different type of normalization were chosen. Next, the best four neural network predictors for every different type of meteorological time-series in order to use them in more complex systems like neural network committee machines were chosen.

Table 1: Comparison between N.N. predictors and ANFIS, which are the correlation coefficient $(\rho)$

\begin{tabular}{lll}
\hline Mean daily solar radiation; & N.N. $\rightarrow 5-15-1$ & $\rho=0.81751$ \\
ANFIS & $\rho=0.81305$ & \\
Mean daily temperature; & N.N. $\rightarrow 5-10-1$ & $\rho=0.97689$ \\
ANFIS & $\rho=0.97699$ & \\
\hline
\end{tabular}

Finally, the best neural network predictor for every different time-series in order to be able to compare its results with ANFIS was chosen. The optimum neural network predictors for every different time-series are introduced.

Daily mean solar radiation: $5-15-1$, normalized in the range 0.1-0.9, using SAF.

Daily mean temperature: $5-10-1$, normalized in the range 0.1-0.9, using SAF.

\section{RESULTS}

The following Figs. 3 and 4 the graphs present: Time plot, error plot and scatter plot. For the training and testing of data a first TS type system consisting of 7 inputs was created. The combination of fuzzy logic with neural networks proved to have very good results in the daily mean solar radiation and temperature forecasting.

Neural network structure for temperature prediction as shown in Table 2, 4-5:

- NNP1: 5-10-1, normalized data in the range 0.1$0.9, \mathrm{SAF}$

- NNP2: 3-5-1, normalized data in the range 0.1-0.9, SAF

- $\quad$ NNP3: 7-3-1, real data, SAF

NNP4: 7-3-1, normalized data in the range 0.1-0.9, SAF

Table 2: Daily mean temperature

\begin{tabular}{lllll}
\hline $\begin{array}{l}\text { Neural } \\
\text { Network }\end{array}$ & $\begin{array}{l}\text { Activation } \\
\text { function }\end{array}$ & MSE & AME & $\rho$ \\
\hline $5-10-1$ & Sigmoid & 0.000801 & 0.020861 & 0.97689 \\
\hline
\end{tabular}

Table 3: Daily mean solar radiation

\begin{tabular}{lllll}
\hline $\begin{array}{l}\text { Neural } \\
\text { Network }\end{array}$ & $\begin{array}{l}\text { Activation } \\
\text { function }\end{array}$ & MSE & AME & $\rho$ \\
\hline $5-15-1$ & Sigmoid & 0.010451 & 0.078213 & 0.8267 \\
\hline
\end{tabular}

Table 4: Values of M.S.E., A.M.E. and $\rho$ for Mean Daily Solar radiation and Mean Daily Temperature;

\begin{tabular}{lllll}
\hline Mean daily & Performance index & MSE & AME & $\rho$ \\
\hline Solar radiation & Committee machine & 0.01104 & 0.06954 & 0.82541 \\
Temperature; & Committee machine & 0.000702 & 0.0209 & 0.97689 \\
\hline
\end{tabular}

Table 5: A Comparison of FP, NNP, ANFISP and CMP predictors;

Forecasting system Performance index $(\rho)$

Daily mean solar radiation

N.N. $\rightarrow 5-15-1 \quad 0.81632$

ANFIS 0.81857

CMP $\quad 0.82245$

Daily mean temperature

$\mathrm{NNP} \rightarrow 5-10-1 \quad 0.9604$

ANFISP $\quad 0.9660$

CMP $\quad 0.9670$

FP 0.9695 


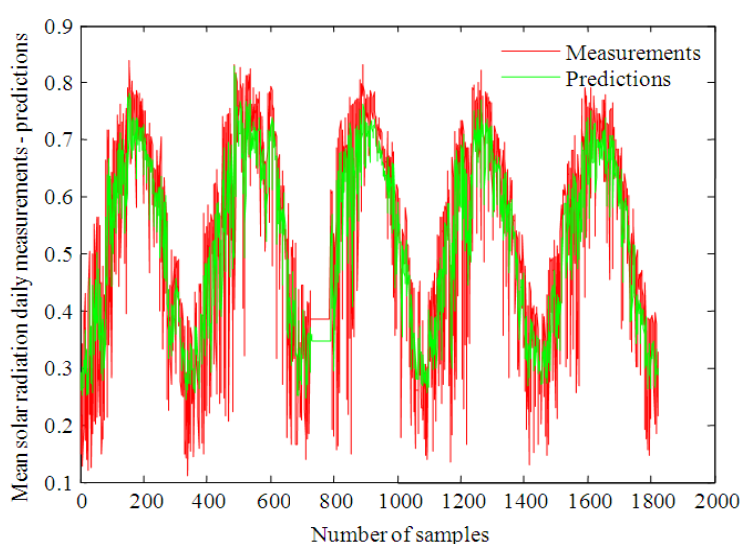

(a)

Fig. 3: Prediction results of temperature with ANFIS

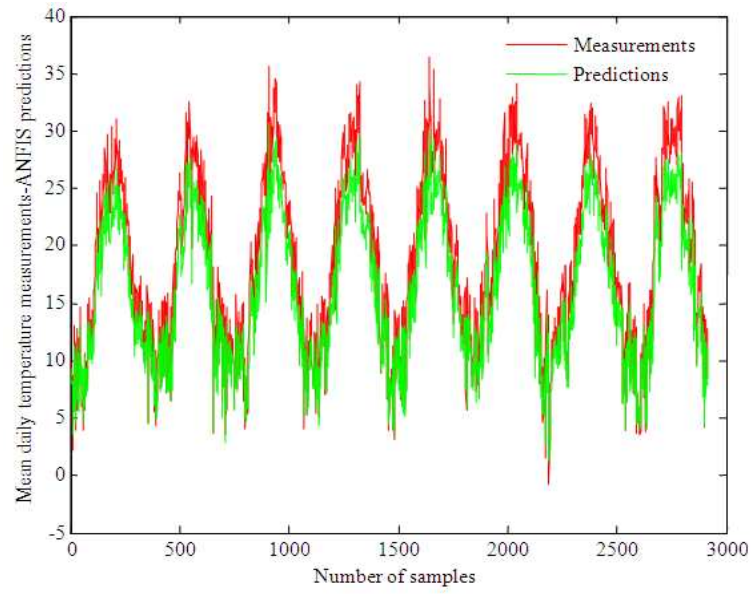

(a)

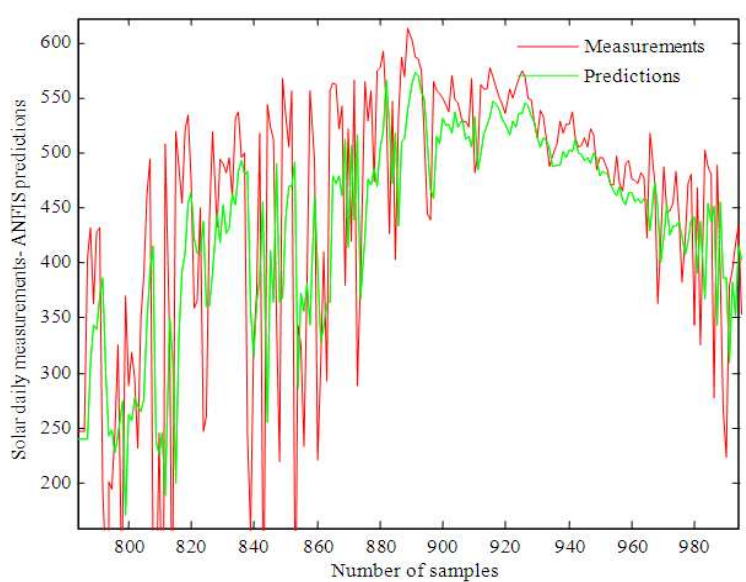

(b)

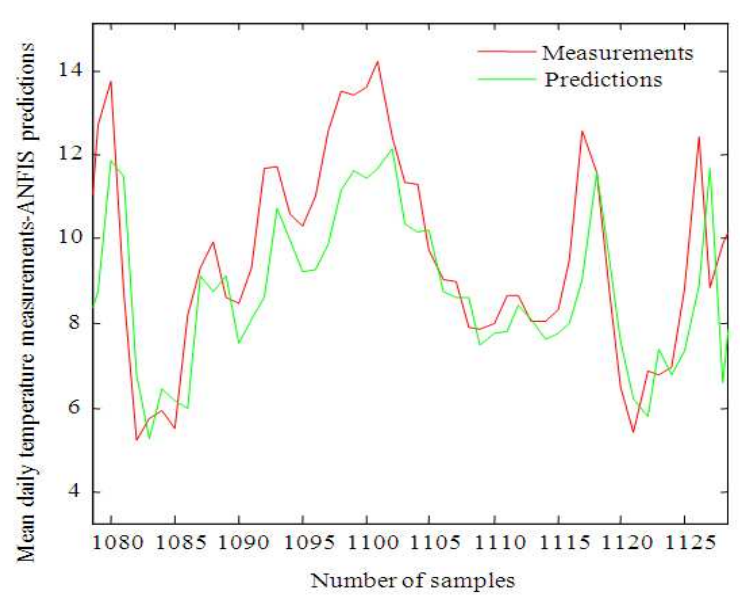

(b)

Fig. 4: Prediction results of temperature with ANFIS for a small sample

\section{DISCUSSION}

Neural network structure for solar radiation prediction as shown in Table 3-5:

- NNP1: 5-15-1, normalized data in the range 0.10.9, SAF

- NNP2: 7-3-1, normalized data in the range 0.1-0.9, SAF

- NNP3: 7-10-1, normalized data in the range 0.10.9, SAF

- NNP4: 5-10-1, normalized data in the range 0.10.9, SAF

The prediction results of the identified TS fuzzy model are compared with those obtained using a linear model as shown in Fig. 5. Contrary to the TS fuzzy models which suppose that the observed time series result from a determinist process.

The linear model supposes that the time series is a signal generated by a linear stochastic process AutoRegressive Moving Average stochastic process (ARMA). To identify the linear model ARMA able to describe the daily solar radiation and the parameters are estimated using. The derived linear regression model (Auto-Regressive (AR) of order two AR (2)):

$\mathrm{y}_{\mathrm{t}}=0.31 \mathrm{y}_{\mathrm{t}-1}-0.021 \mathrm{y}_{\mathrm{t}-2}+\varepsilon_{\mathrm{t}}$

where, $\varepsilon_{\mathrm{t}}$ is a white noise with mean 0 and variance $\sigma_{2}=$ 0.824 . It can be observed, according to the Table 5, that MSE found are 2.974, 3.2924 respectively for train and test. The linear models are represents less than 8 and $11 \%$ of the mean of the daily solar radiation data. 


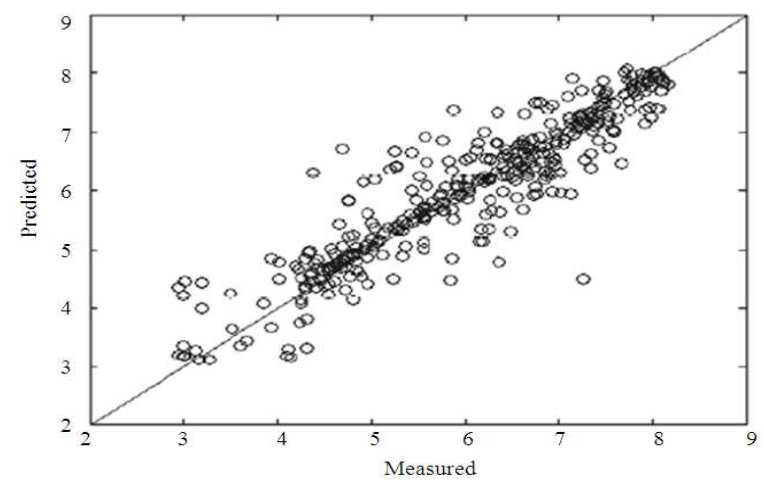

(a)

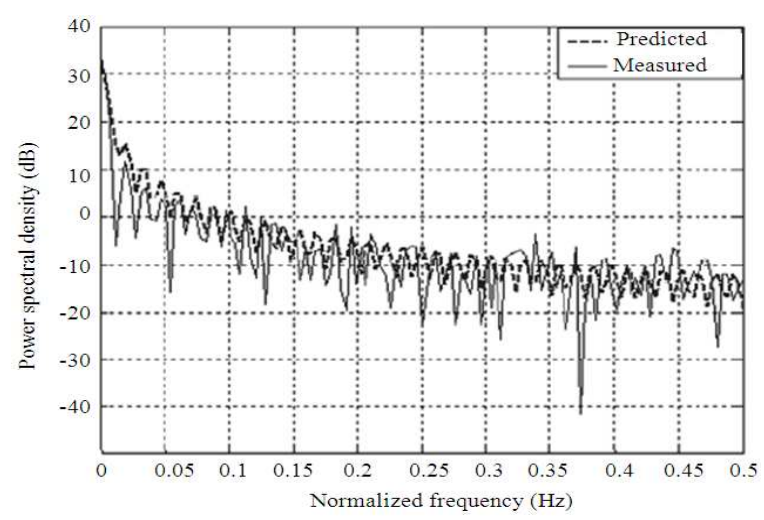

(b)

Fig. 5: Scatter diagram, spectra of measured and predicted mean daily solar radiation

The adequacy of fit is also assessed using the agreement index, which returns the percentage of similarity between the measured and the estimated data of the daily solar radiation. We obtain $96 \%$ in the case of the TS fuzzy model and $82 \%$ for the linear model. These results are very satisfactory and the model is considered adequate for such predictions, which strengthens the robustness of the TS fuzzy model.

\section{CONCLUSION}

Concerning neuron-fuzzy predictors and more specifically ANFIS it is proved that the combination of linguistic rules of fuzzy logic with the training algorithm used in neural networks, contribute in very qualitative prediction results, which approach the 'best' neural predictor's results. The structure of the TS fuzzy model is identified using a method which permits to determine the optimal structure on automatic manner while being based on the calculation of the importance of the rules in the calculation of the model output. However, the linear parameters are estimated by the WLS algorithm and the non-linear parameters are estimated using the TS method. Additionally, a comparison is performed between the identified TS fuzzy model. The obtained results show that the identified TS fuzzy model provides satisfactory performances. So, we conclude that the fuzzy systems can be used as an alternative method to generate solar data for locations where measurements are not available.

\section{REFERENCES}

Abd Elaal, A.K., H.A. Hefny and A.H. Abd Elwahab, 2010. Constructing fuzzy time series model based on fuzzy clustering for a forecasting. J. Comp. Sci., 6: 735-739. DOI: 10.3844/jcssp.2010.735.739

Aggarwal, K.K., Y. Singh, P. Chandra and M. Puri, 2005. Measurement of software maintainability using a fuzzy model. J. Comp. Sci., 1: 538-542. DOI: 10.3844 /jcssp.2005.538.542

Ahmed, S. and Z. Isa, 2009. Modeling and Forecasting Volatility of the Malaysian Stock Markets. J. Mathematics and Statistics, 5: 234-240. DOI: 10.3844/jmssp.2009.234.240

Alexandros E. Milionis and Hayette Gatfaoui, 2010. Special Issue for the 6th International Conference on Applied Financial Economics, Samos, Greece, 2-4 July 2009. Am. J. Econ. Bus. Admin., 2: 339340. DOI: 10.3844/ajebasp.2010.339.340

Alsaade, F., 2010. A study of neural network and its properties of training and adaptability in enhancing accuracy in a multimodal biometrics scenario. Inform. Technol. J., 9: 188-191.

Al-Suhaibani, S.A., A. A. Al-Janobi and Y. N. AlMajhadi, 2010. Development and evaluation of tractors and tillage implements instrumentation system. Am. J. Eng. Applied Sci., 3: 363-371. DOI: 10.3844/ajeassp.2010.363.371

Anton A. Kamil, Adli Mustafa and Khlipah Ibrahim, 2009. Stochastic optimization for portfolio selection problem with mean absolute negative deviation measure. J. Math. Stat., 5: 379-386. DOI: 10.3844/jmssp.2009.379.386

Babuska, R., 1996. Fuzzy Modellingtnd Identification. Delft University of Technology.

Chen, S.M., 1994. A weighted fuzzy reasoning algorithm for medical diagnosis. Decis. Support Syst., 11: 37-43. DOI:10.1016/01679236(94)90063-9

Dastorani, M.T., A. Talebi and M. Dastorani, 2010. Using neural networks to predict runoff from ungauged catchments. Asian J. Applied Sci., 3: 399-410. 
Efendioglu, A.M. and A. T. Karabulut, 2009. Cceptance and use of strategic processes in developing countries. Am. J. Econom. Business Adminis., 1: 150-159. DOI: 10.3844/ajebasp.2009.150.159

Effendi, Z. R. Ramli and J. A. Ghani, 2010. A back propagation neural networks for grading jatropha curcas fruits maturitiy. Am. J. Applied Sci., 7: 390-394.

Eslamian, S.S., J. Abedi-Koupai, M.J. Amiri and S.A. Gohari, 2009. Estimation of daily reference evapotranspiration using support vector machines and artificial neural networks in greenhouse. Res. J. Enviorn. Sci., 3: 439-447.

Guang, L., W. Ya-Dong and S. Xiao-Hong, 2009. A privacy preserving neural network learning algorithm for horizontally partitioned databases. Inf. Technol. J., 9: 1-10.

Huarng, K.H., T.H.K. Yu and Y.W. Hsu, 2007. A multivariate heuristic model for fuzzy time-series forecasting. IEEE Trans. Syst. Man and Cybernetics-Part B: Cybernetics, 37: 836-846. DOI: 10.1109/TSMCB.2006.890303

Ismail, A. R., Y.M. Yusof, N. K. Makhtar, B. M. Deros and M. R.A. Rani, 2010. Optimization of temperature level to enhance worker performance in automotive industry. Am. J. Applied Sci., 7: 360-365. DOI: 10.3844/ajassp.2010.360.365

Kang, P. and Z. Jin, 2010. Neural network sliding mode based current decoupled control for induction motor drive. Inf. Technol. J., 9: 1440-1448.

Kar, A. and S. Kundu, 2010. Odyssey in Calcutta. Am. J. Econ. Bus. Admini., 2: 136-140. DOI: 10.3844/ajebasp.2010.136.140

Li, S.T. and Y.C. Cheng, 2009. An enhanced deterministic fuzzy time series forecasting model. cybernetics and systems. Int. J., 40: 211-235. DOI: 10.1080/01969720802715128
Mahi, H. and H.F. Izabatene, 2011. Segmentation of satellite imagery using RBF neural network and genetic algorithm. Asian J. Applied Sci., 4: 186194. DOI: 10.3923/ajaps.2011.186.194

Michael Wagner, 2010. Forecasting Daily Demand in Cash Supply Chains. American Journal of Economics and Business Administration, 2: 377383. DOI: 10.3844/ajebasp.2010.377.383

Podeh, S.S., J. Oladi, M.R. Pormajidian and M.M. Zadeh, 2009. Forest change detection in the north of iran using TM/ETM+Imagery. Asian J. Applied Sci., 2: 464-474. DOI: 10.3923/ajaps.2009.464.474

Pratumsuwan, P., S. Thongchai and S. Tansriwong, 2010. A hybrid of fuzzy and proportional-integralderivative controller for electro-hydraulic position servo system. Energy Res. J., 1: 62-67. DOI: 10.3844/erjsp.2010.62.67

Saleh, A., T. Tsuji and T. Oyama, 2009. optimal bidding strategies for generation companies in a day-ahead electricity market with risk management taken into account . Am. J. Eng. Applied Sci., 2: 816. DOI: 10.3844/ajeassp.2009.8.16

Solaimani, K., 2009. A study of rainfall forecasting models based on artificial neural network. Asian J. Applied Sci., 2: 486-498. DOI: 10.3923/ajaps.2009.486.498

Yedjour, H., B. Meftah, D. Yedjour and A. Benyettou, 2011. Combining spiking neural network with hausdorff distance matching for object tracking. Asian J. Applied Sci., 4: 63-71. DOI: 10.3923/ajaps.2011.63.71 